\title{
Long non-coding RNA GHET1 promotes thyroid cancer cell proliferation and invasion
}

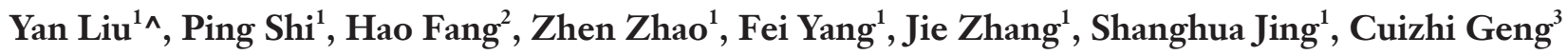 \\ ${ }^{1}$ Department of Otorhinolaryngology, Hebei Medical University Fourth Affiliated Hospital and Hebei Provincial Tumor Hospital, Shijiazhuang, \\ China; ${ }^{2}$ Hepatobiliary Surgery Department, Hebei Medical University Fourth Affiliated Hospital and Hebei Provincial Tumor Hospital, \\ Shijiazhuang, China; ${ }^{3}$ Breast Oncology Department, Hebei Medical University Fourth Affiliated Hospital and Hebei Provincial Tumor Hospital, \\ Shijiazhuang, China \\ Contributions: (I) Conception and design: Y Liu; (II) Administrative support: C Geng; (III) Provision of study materials or patients: P Shi, H Fang; (IV) \\ Collection and assembly of data: F Yang, Z Zhen; (V) Data analysis and interpretation: S Jing, J Zhang; (VI) Manuscript writing: All authors; (VII) \\ Final approval of manuscript: All authors. \\ Correspondence to: Cuizhi Geng. Breast Oncology Department, Hebei Medical University Fourth Affiliated Hospital and Hebei Provincial Tumor \\ Hospital, 12 Jiankang Road, Chang'an District, Shijiazhuang 050000, China. Email: geng0923@163.com.
}

Background: We aimed to evaluate the role of long non-coding RNA (LncRNA) gastric carcinoma proliferation-enhancing transcript 1 (GHET1) on thyroid cancer (TC) behavior in vitro.

Methods: TC tissues and paired adjacent normal tissues were obtained after surgical excision from 43 patients with TC. The expression of LncRNA GHET1 was analyzed by real-time (RT) PCR. Human papillary thyroid cancer cell lines (TPC-1, BCPAP) were used to examine the role of LncRNA GHET1 in vitro. Cell proliferation was determined by CCK8 and cell colony formation assays. Transwell and woundhealing assays were used to detect the invasion and migration of thyroid cancer cells.

Results: Our results showed that LncRNA GHET1 was significantly more upregulated in TC tissues than in adjacent normal tissues. LncRNA GHET1 was also increased in thyroid cancer cell lines compared to normal thyroid cell lines. The upregulation of LncRNA GHET1 was significantly associated with tumor invasion, gender, and lymph node metastasis in patients with thyroid cancer. The in vitro studies showed that silencing LncRNA GHET1 in BCPAP cells inhibited cell proliferation, cell invasion, and migration. Silencing of LncRNA GHETI also promoted the cell apoptotic rate, caused an increase in the cell population at the G0/G1 phase, and decreased the cell population at the $\mathrm{S}$ phase. In contrast, the overexpression of LncRNA GHET1 promoted cell proliferation, invasion, and migration, inhibited cell apoptosis, and increased cell population at the $\mathrm{S}$ phase in TPC cells.

Conclusions: LncRNA GHET1 dysregulation might be involved in the carcinogenesis of thyroid cancer. LncRNA GHET1 could be used as a potential molecular marker and molecular target for TC.

Keywords: Thyroid cancer; LncRNA GHET1; proliferation; invasion and migration; apoptosis

Submitted Jul 16, 2021. Accepted for publication Sep 07, 2021.

doi: $10.21037 /$ tcr-21-1615

View this article at: https://dx.doi.org/10.21037/tcr-21-1615

\footnotetext{
$\wedge$ ORCID: 0000-0001-6646-6215.
} 


\section{Introduction}

The incidence of thyroid carcinoma (TC), which usually originates from follicular or parafollicular thyroid cells, has shown a rapid increase over the past two decades $(1,2)$. The number of global deaths from TC has increased from 24,000 in 1990 to 36,000 in 2010 (3). In addition, the age at onset has drastically decreased (4). Although papillary thyroid carcinoma (PTC) exhibits a satisfactory outcome after surgery or radioiodine treatment, approximately $15 \%$ of patients relapse and acquire distant metastases (5). In addition, the underlying molecular mechanisms involved in TC carcinogenesis are still not fully understood.

Long noncoding RNAs (LncRNAs) are transcribed RNAs, more than 200 nucleotides in length, that do not translate proteins (6). LncRNAs have been linked to every stage of cell life, including cell proliferation, differentiation, apoptosis, and cell motility (7). A global network-based strategy, BiRWLGO, is a relatively efficient method used to predict the functions of LncRNAs (8). A number of studies have demonstrated that dysregulated LncRNAs are associated with the tumorigenesis and progression of human cancers $(5,6,9,10)$. Among the cancer-related LncRNAs, gastric carcinoma proliferation-enhancing transcript 1 (GHET1) exhibits high efficiency, stability, and specificity and shows great promise in the treatment of cancer (11). GHET1 is highly expressed in diverse malignancies. Furthermore, GHET1 performs key functions in carcinogenesis and progression, suggesting that GHET1 is expected to be a prospective biomarker or therapeutic target for cancers. The GHET1 gene was first identified by Yang et al. (12) in gastric cancer tissues in 2014; it contains 1,913 nucleotides and is located at chromosome $7 \mathrm{q} 36.1$. LncRNA GHET1 has been closely correlated with tumor size, invasion, and migration (13). Li et al. have proved that LncRNA GHET1 plays an important role in regulating osteoblast differentiation and proliferation, which may be mentioned by the PTEN signaling pathway (14). LncRNA GHET1 functions as an oncogene in several cancers, yet its role in thyroid cancer has not been investigated to date (15).

This study aimed to examine the biological role of LncRNA GHET1 in thyroid cancer. We present the following article in accordance with the MDAR reporting checklist (available at https://dx.doi.org/10.21037/tcr-21-1615).

\section{Methods}

\section{Clinical patient data and tissue samples}

A total of 43 patients with TC treated at the Hebei Provincial Tumor Hospital between January 2018 and May 2019 were enrolled in the present study. None of the subjects received radiation or chemotherapy therapy before surgery. All normal tissues and paired TC tissues were obtained from thyroidectomy. After surgery resection, the tumor tissues and adjacent normal tissues were kept in RNAlater Stabilization Solution, after which samples were stored at $4{ }^{\circ} \mathrm{C}$ overnight and then below $-80{ }^{\circ} \mathrm{C}$ until further use. Adjacent normal tissues were taken $>3 \mathrm{~cm}$ from the cancer tissues.

All patients provided informed written consent, and this study was approved by the Ethics Committee of the Hebei Provincial Tumor Hospital (Ethics Review Number: 2020KS017). All procedures performed in this study involving human participants were in accordance with the Declaration of Helsinki (as revised in 2013).

\section{Cell culture}

The human thyroid cancer cell lines (TPC-1, BCPAP) and the normal thyroid cell line (Nthy-ori3-1) were provided by Shanghai Zhong Qiao Xin Zhou Biotechnology Company. All cells were cultured in Dulbecco's Modified Eagle's Medium (DMEM; Thermo Fisher Scientific, Waltham, MA, USA) supplemented with $10 \%$ fetal bovine serum (FBS) and 1\% penicillin/streptomycin (all from Gibco; Thermo Fisher Scientific, Inc.) at $37{ }^{\circ} \mathrm{C}$ in a humidified incubator with $5 \% \mathrm{CO}_{2}$. Nthy-ori3-1 is the normal control.

\section{Cell transfection with plasmid and small interfering $R N A s$}

LncRNA GHET1 was knocked down by specific small interfering RNAs. The siRNAs for GHET1 (si-GHET\#1: 5'-GAGAAAUAGUCUGUGUUGCCCUGAA-3' and si-GHET1\#2: 5'-CGGCAGGCAUUAGAGAUG AACAGCA-3') and the scrambled siRNA (si-NC; siNC, 5'-UUCUCCGAACGUGUCACGUTT-3') were purchased from RiboBio (Guangzhou, China). The LncRNA GHET1 overexpression plasmid was constructed by inserting the amplified LncRNA GHET1 cDNA into a pcDNA3.1 vector obtained from GenePharma (Shanghai, 
China).

The TPC and BCPAP cells were grown in six-well plates until they reached $70 \%$ confluence. The cells were then transfected with plasmids using Lipofectamine 2000 (Invitrogen, Carlsbad, CA, USA) and FuGENE HD Transfection Reagent (Promega Corporation, USA) according to the manufacturer's instructions. The expression of LncRNA GHET1 was detected by real-time PCR.

\section{qRT-PCR}

The RNAs obtained from the thyroid cancer cells were extracted using TRIzol reagent (Thermo Fisher Scientific, Inc., USA). Subsequently, the RNA was reverse transcribed into complementary DNA (cDNA) using a Reverse Transcription System kit (Promega Corporation, USA). Then, qPCR was performed with an ABI 7300 Real-Time PCR System (Applied Biosystems; Thermo Fisher Scientific, Inc.) by applying a Power SYBR Green Master Mix Kit (Thermo Fisher Scientific Inc, Waltham, MA, USA). GAPDH was used as the reference. The calculation of gene expressions was based on the $2^{-\Delta \Delta C t}$ method. The primers for LncRNA GHET1 were as follows: forward 5'-TTCCCAACACCCTATAAGAT-3' and reverse 5'-CCCCACAAATGAAGACACT-3'. The primers for GAPDH were as follows: forward 5'-TTCTAGACGGCAGGTCAGG-3' and reverse 5'-ACCCAGAAGACTGTGGATGG-3'.

\section{Cell counting kit-8 (CCK-8) assay}

The cell viability was examined using a Cell Counting Kit-8 (CCK8, R\&S Biotechnology, Shanghai, China) reagent. In short, TPC and BCPAP cells were plated into 96-well plates $\left(1 \times 10^{3} /\right.$ well). After $0,24,48$, and $72 \mathrm{~h}$, each well was mixed with a CCK-8 solution $(10 \mu \mathrm{L})$ and incubated at $37{ }^{\circ} \mathrm{C}$ for an additional $2 \mathrm{~h}$. A microplate absorbance reader (BioRad Laboratories, CA) was used to measure the absorbance (wavelength: $450 \mathrm{~nm}$ ).

\section{Cell colony formation assay}

For the colony formation assay, TPC and BCPAP cells were seeded into 6-well plates. The cell density was 200 cells/well. The cells were then transfected with either si-GHET1\#2, si-NC, pcDNA-GHET1, or pcDNA and cultured for 14 days in a DMEM medium containing $10 \%$ FBS. The medium was replaced with fresh medium every 3 days. The si-NC and the pcDNA are the normal control for each experiment. Cells were then fixed with $4 \%$ paraformaldehyde and stained with $1 \%$ crystal violet for 20 min, after which the colonies were measured.

\section{Transwell assay}

After transfection, TPC and BCPAP cells were cultured in a serum-free culture medium for 2 days. For the migration assay, the cells were placed in a higher chamber of the insert (Millipore, USA). The pore size was $8 \mu \mathrm{m}$. The cells were then transfected with either si-NC, si-GHET1\#2, pcDNA, or pcDNA-GHET1. The different groups of cells were collected and suspended in a non-bovine serum medium. The cell density was $2 \times 10^{5}$ cells $/ \mathrm{mL}$. Subsequently, $200 \mu \mathrm{L}$ of the suspended cell solution was added to the upper chamber of the transwell membrane, while the media with $10 \%$ FBS was added to the lower chamber. After $24 \mathrm{~h}$, the non-migrating cells were removed with a cotton swab, and $4 \%$ paraformaldehyde was used to fix the migrating cells, which were stained with $0.1 \%$ crystal violet for twenty min at $25^{\circ} \mathrm{C}$. The migrated cells were counted in five random visual fields and then imaged using a light microscope (Olympus Corporation, Japan). The invasion assay was performed according to the same method used for the migration assay except that the Transwell insert chambers were coated with Matrigel (BD Biosciences).

\section{Wound-bealing assays}

The TPC and BCPAP cells were seeded in different plates. Then the cells were transfected with pcDNA and pcDNAGHET1. After $6 \mathrm{~h}$, a scratch was made in the plates using a $10-\mu \mathrm{L}$ pipette tip held perpendicular to the plane. The residual cells were washed with $\mathrm{PBS} 3-5$ times, and the culture medium was replaced. The cells in the different groups were observed and photographed at 0 and $48 \mathrm{~h}$ using a microscope. Remodeling was measured as the diminishing distance across the induced injury, normalized to the $0 \mathrm{~h}$ control, and expressed as the relative migration. The experiment was repeated three times, and the average value was taken.

\section{Flow cytometry}

\section{Cell apoptosis}

The TPC and BCPAP cells were washed twice using PBS adjusted to a concentration gradient of $1 \times 10^{6} / \mathrm{mL}$. Then, 
Table 1 Clinical characteristics

\begin{tabular}{|c|c|c|c|c|}
\hline \multirow[b]{2}{*}{ Variable } & \multicolumn{2}{|c|}{ GHET1 expression } & \multirow[b]{2}{*}{$\chi^{2}$} & \multirow{2}{*}{$\begin{array}{c}\mathrm{P} \\
\text { value }\end{array}$} \\
\hline & $\begin{array}{c}\text { Low } \\
\text { expression }\end{array}$ & $\begin{array}{c}\text { High } \\
\text { expression }\end{array}$ & & \\
\hline Age (years) & & & 0.007 & 0.933 \\
\hline$<55$ & 16 & 17 & & \\
\hline$\geq 55$ & 5 & 5 & & \\
\hline Gender & & & 3.995 & 0.046 \\
\hline Male & 6 & 1 & & \\
\hline Female & 16 & 20 & & \\
\hline Tumor number & & & 0.573 & 0.449 \\
\hline Solitary & 16 & 13 & & \\
\hline Multiple & 6 & 8 & & \\
\hline Tumor size (cm) & & & 0.77 & 0.781 \\
\hline$<1$ & 13 & 15 & & \\
\hline$\geq 1$ & 5 & 7 & & \\
\hline $\begin{array}{l}\text { Tumor invasion } \\
\text { (TNM stage) }\end{array}$ & & & 5.795 & 0.016 \\
\hline $\mathrm{T} 1-\mathrm{T} 2$ & 17 & 10 & & \\
\hline T3-T4 & 4 & 12 & & \\
\hline $\begin{array}{l}\text { Lymph node } \\
\text { metastasis }\end{array}$ & & & 5.464 & 0.019 \\
\hline No & 12 & 12 & & \\
\hline $\mathrm{N} 1$ & 3 & 16 & & \\
\hline
\end{tabular}

GHET1, gastric carcinoma proliferation-enhancing transcript 1; TNM, tumor, node, metastasis.

a total amount of $100 \mu \mathrm{L}$ cell suspension was transferred to a $5-\mathrm{mL}$ flow tube, followed by the respective additions of $10 \mu \mathrm{L}$ of propidium iodide (PI) (BD Biosciences, USA) and $5 \mu \mathrm{L}$ of annexin V-FITC and then gently agitated. The cells were incubated at $25^{\circ} \mathrm{C}$ for $15 \mathrm{~min}$. Before the flow cytometry analysis (FC500 type; Beckman Coulter), $400 \mu \mathrm{L}$ of binding buffer was added. This experiment was run in triplicate.

\section{Cell cycle}

The TPC and BCPAP cells were washed three times with PBS before being fixed in $70 \%$ ice-cold ethanol at $4{ }^{\circ} \mathrm{C}$ for $6 \mathrm{~h}$. Then the ethanol was removed. The cells were cleaned with PBS and then digested using RNAse. Subsequently, $50 \mu \mathrm{g} / \mathrm{mL}$ propidium iodide was added to the cell suspension. DNA staining was performed for $30 \mathrm{~min}$, and then the distribution of DNA content was analyzed by flow cytometry. Multicycle AV software was used to calculate the percentage of each cell cycle phase. This experiment was run in triplicate.

\section{Statistical analysis}

The data analysis was performed with SPSS 22.0 software (SPSS Inc., Chicago, IL, USA). The data are presented as the mean \pm standard deviation and were analyzed using the Student's $t$-test (for two-group comparisons) or oneway ANOVA (for multiple group comparisons). A Chisquare test was used to analyze the association between the GHET1 expression and clinicopathological parameters. All statistical tests were two-sided, and a $P$ value $\leq 0.05$ was considered statistically significant.

\section{Results}

\section{LncRNA GHET1 is overexpressed in TC tissues and is associated with clinicopathological features}

The age range of the 43 thyroid cancer patients was 13-64 years, with a median age of 37 years (21 patients were $>40$ years old, and 22 patients were $<37$ years old). There were 27 cases of T1-T2 and 16 cases of T3-T4. Fortytwo patients had PTC, and one case had medullary thyroid carcinoma; lymph node metastasis was found in 19 cases, while the remaining cases [24] were without lymph node metastasis. Table 1 shows the clinical information for all patients.

We evaluated the association between clinicopathological features and LncRNA GHET1 expression levels in TC tissues. As shown in Table 1, a high expression of LncRNA GHET1 was significantly correlated with tumor invasion $(\mathrm{P}=0.016)$, gender $(\mathrm{P}=0.046)$, and lymph node metastasis $(\mathrm{P}=0.019)$.

QRT-PCR analyses revealed that LncRNA GHET1 expression was significantly upregulated in TC tissues compared with adjacent normal tissues $(\mathrm{P}<0.01$, Figure $1 A)$. LncRNA GHET1 expression was assessed in the TC cell lines (TPC and BCPAP) and the normal thyroid cell line (Nthy-ori3-1). LncRNA GHET1 expression was significantly higher in the TC cell lines than in the normal thyroid cell line $(\mathrm{P}<0.01$, Figure $1 B)$. Moreover, LncRNA GHET1 expression was relatively higher in BCPAP cells. Consequently, we chose BCPAP cells for transfection with 
A

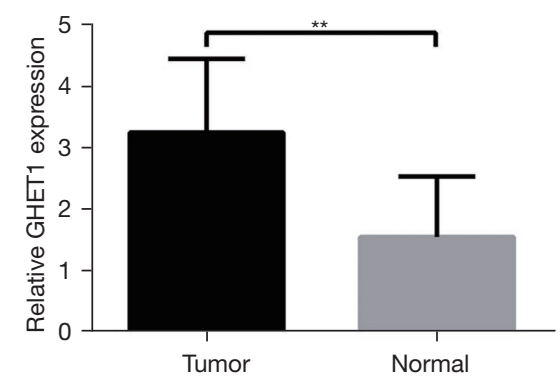

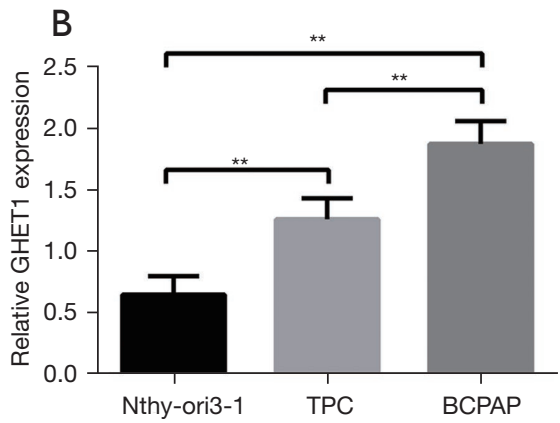

Figure 1 LncRNA GHET1's expression in tissues thyroid cancer cell lines and. (A) QRT-PCR results showing the expression of LncRNA GHET1 in the TC tissues and adjacent non-tumor tissues of 43 thyroid cancer patients. (B) Expression of LncRNA GHET1 in two thyroid cancer cell lines (TPC, BCPAP) and normal human thyroid cell line (Nthy-ori3-1) detected by qRT-PCR. Data are presented as mean \pm SD. **, $\mathrm{P}<0.0001$ vs. respective controls.

siNC and siGHET1.

\section{Silencing LncRNA GHET1 suppresses TC cell proliferation, invasion and migration, and promotes cell apoptosis and cell cycle arrest in vitro}

To evaluate the effects of LncRNA GHET1 knockdown in TC cells, we transfected BCPAP cells with si-NC, siGHET1\#1, and si-GHET1\#2. QRT-PCR results showed that after transfection with si-GHET1\# and si-GHET1\#2, the expression of LncRNA GHET1 was significantly downregulated in BCPAP cells $(\mathrm{P}<0.01)$ compared to those transfected with si-NC (Figure $2 A$ ). As si-GHET1\#2 had more profound effects on suppressing LncRNA GHET1 expression, si-GHET1\#2 was used for further experiments.

The proliferation rates of the BCPAP cells transfected with either non-specific siRNA or si-GHET1 were measured by a CCK- 8 assay. The CCK- 8 assay revealed that, compared with the NC groups, knockdown of LncRNA GHET1 suppressed BCPAP cell proliferation at 24,48 , and $72 \mathrm{~h}$ post-transfection (all $\mathrm{P}<0.05$; Figure $2 B$ ). Furthermore, LncRNA GHET1 knockdown also markedly suppressed the number of colonies in BCPAP cells (Figure 2C). To sum up, silencing LncRNA GHET1 could suppress the proliferation of thyroid cancer.

Next, by using flow cytometry, we verified the effect of LncRNA GHET1 on cell cycle and apoptosis in thyroid cancer cells. Results showed that LncRNA GHET1 knockdown in thyroid cancer cell lines increased the percentage of apoptotic cells compared to the normal group $(\mathrm{P}<0.05$, Figure $2 D)$. In the cell cycle analysis, LncRNA GHET1 downregulation in BCPAP cells increased the cell population at the $\mathrm{G} 0 / \mathrm{G} 1$ phase $(\mathrm{P}<0.05)$ and decreased the cell population at the $\mathrm{S}$ phase $(\mathrm{P}<0.05$, Figure $2 E)$. These data further suggested that silencing LncRNA GHET1 could induce cell cycle arrest in vitro and promote apoptosis.

Furthermore, we determined the role of LncRNA GHET1 on TC cell migration and invasion by using Transwell analyses. Transwell assay revealed that the number of invasive BCPAP cells was significantly lower in the si-GHET1\#2 groups than in the $\mathrm{NC}$ groups $(\mathrm{P}<0.05$, Figure $2 F$ ). These results showed that silencing LncRNA GHET1 could suppress the migration and invasion of thyroid cancer cells.

\section{Overexpression of LncRNA GHET1 promotes TC cell invasion, migration, proliferation, and cell cycle progression and inbibits cell apoptosis}

To study the overexpression effects of LncRNA GHET1 in TC cells, we used TPC cell lines and transfected them with pcDNA-GHET1 and pcDNA. The transfection with pcDNA-GHET1 increased LncRNA GHET1 expression in TPC cells $(\mathrm{P}<0.05$, Figure $3 A)$. The overexpression of LncRNA GHET1 significantly promoted TPC cell proliferation $(\mathrm{P}<0.05$, Figure $3 B)$. Similarly, colony formation showed that LncRNA GHET1 overexpression increased the number of colonies $(\mathrm{P}<0.05$, Figure 3C). Flow cytometry showed that overexpression of LncRNA GHET1 significantly decreased cell population at the G0/G1 phase, suppressed cell apoptosis, and increased cell population at the S phase (Figure 3D,3E). Furthermore, the Transwell assay results showed that LncRNA GHET1 overexpression dramatically increased the migratory and invasive abilities 
A

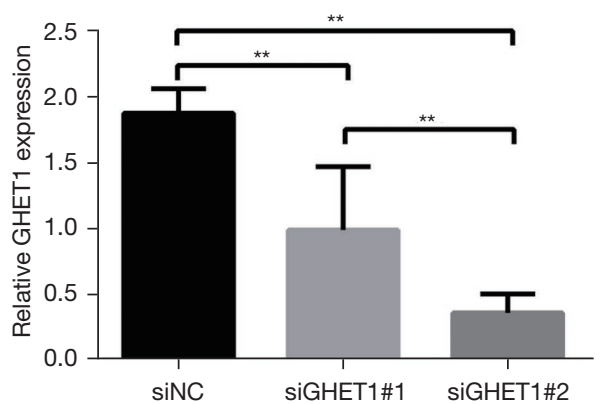

C
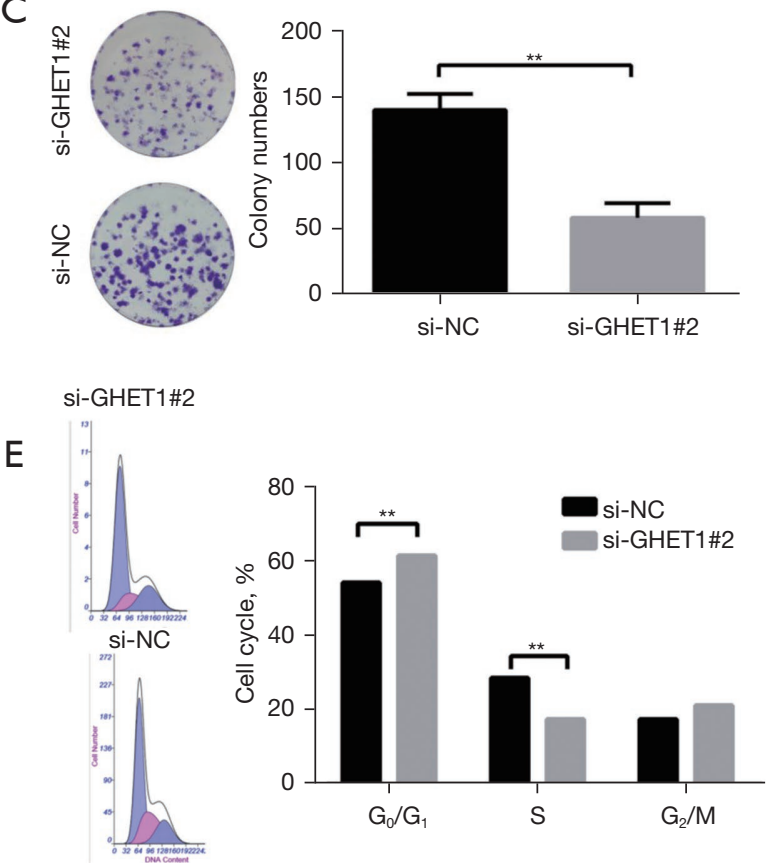

B

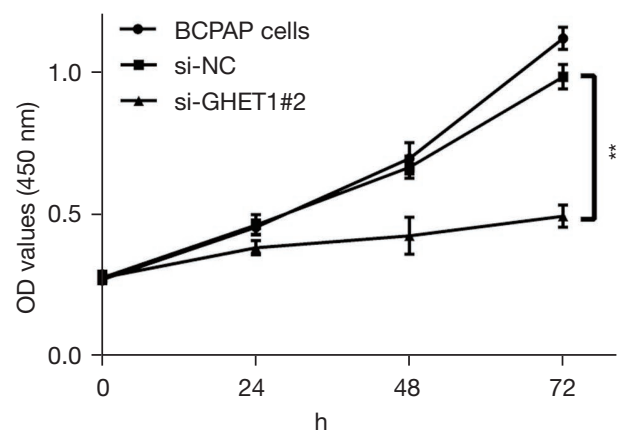

D
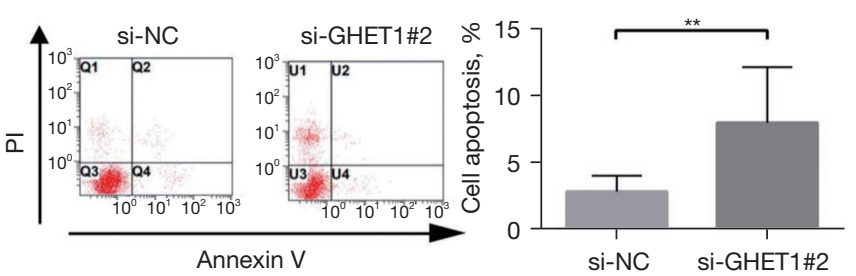

$\mathrm{F}$
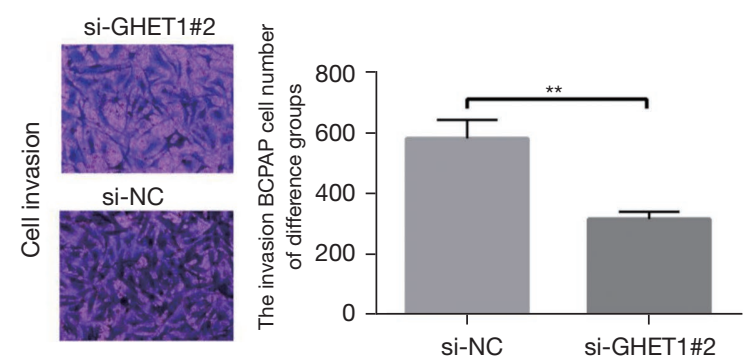

Figure 2 The effects of LncRNA GHET1 knockdown on BCPAP cell migration, apoptosis, , invasion cell cycle, and proliferation. (A) QRT-PCR results showing LncRNA GHET1 expression in BCPAP cells transfected with si-NC, si-GHET1\#1, and si-GHET\#2. (B) CCK8 showing the influence of LncRNA GHET1 knockdown on BCPAP cell proliferation. (C) Colony formation assay results showing the influence of LncRNA GHET1 knockdown on BCPAP proliferation. Crystal violet staining. (D) Cell apoptosis and (E) cell cycle determined by flow cytometry. (F) The Transwell assay showing the influence of LncRNA GHET1 knockdown on BCPAP cell invasion. Crystal violet staining, $\times 200 .{ }^{* *}, \mathrm{P}<0.05$.

of TPC cells $(\mathrm{P}<0.05$, Figure $3 F)$. Wound-healing assays showed that the wound healing rate was markedly inhibited in the control groups (all $\mathrm{P}<0.05$, Figure $3 G$ ).

\section{Discussion}

LncRNAs may act either as tumor suppressor genes or oncogenes. LncRNAs have multiple target genes, and their role in tumor growth and differentiation may vary among individuals and organs (16). The activities of LncRNAs can be associated with miRNAs; LncRNAs may be the precursors of miRNAs $(2,17,18)$.

LncRNA GHET1 has been considered a promising oncogenic LncRNA owing to its high stability, efficiency, and specificity in various types of human cancer. A recent study indicated that an increased LncRNA GHET1 expression level is associated with poor OS, tumor progression, and TNM stage in patients with multiple 
A

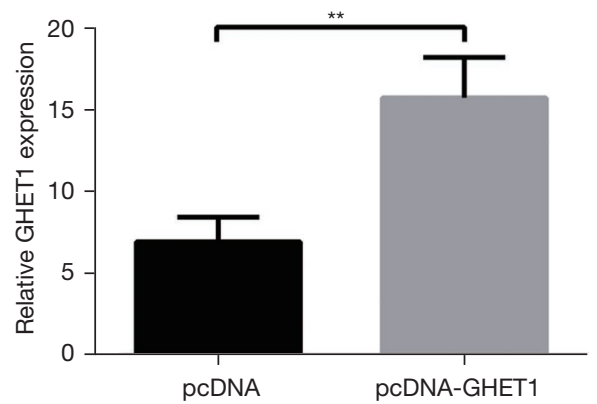

C

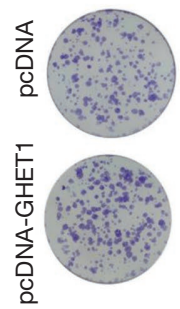

E
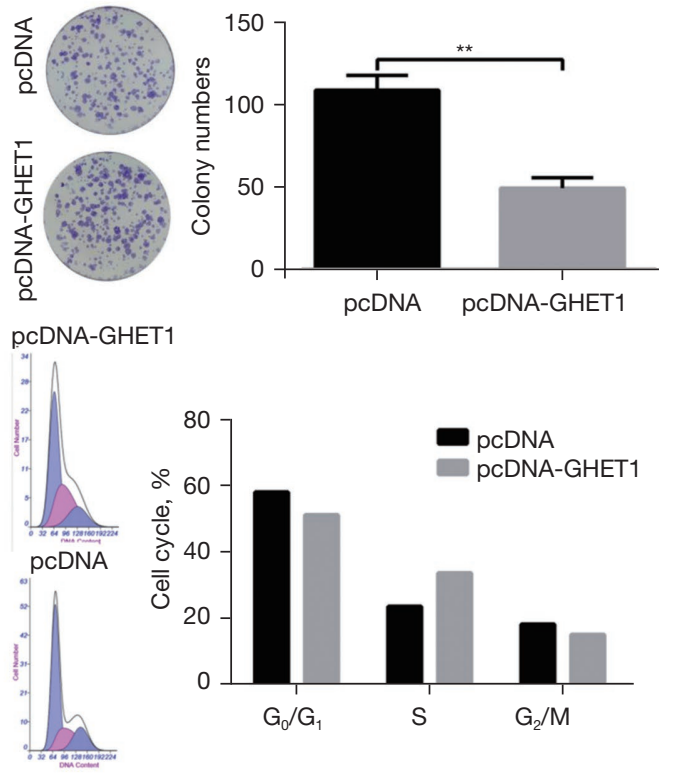

D
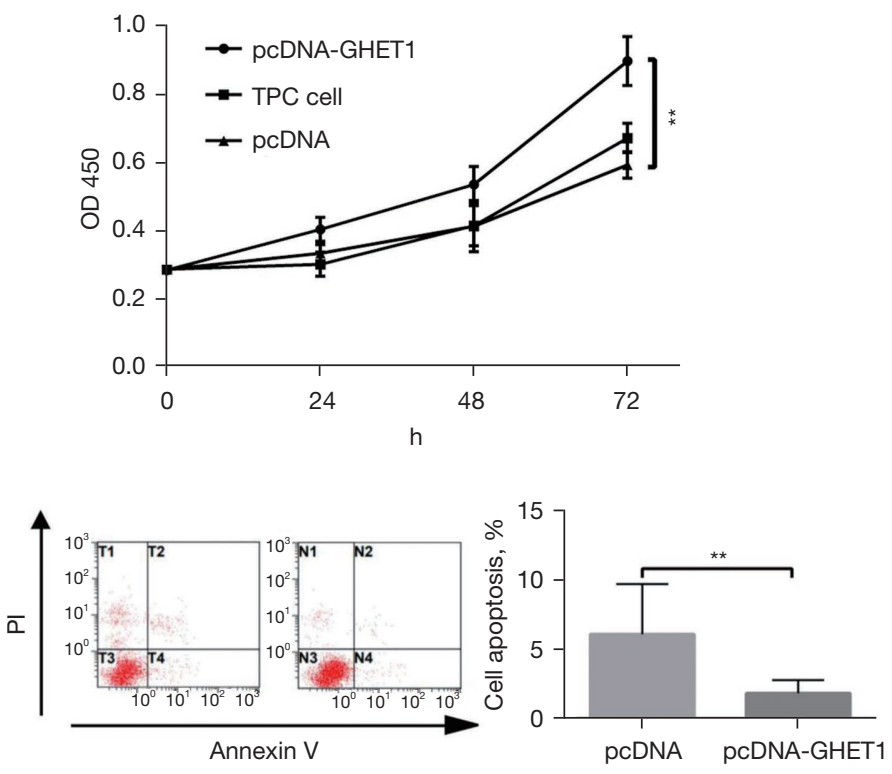

$\mathrm{F}$

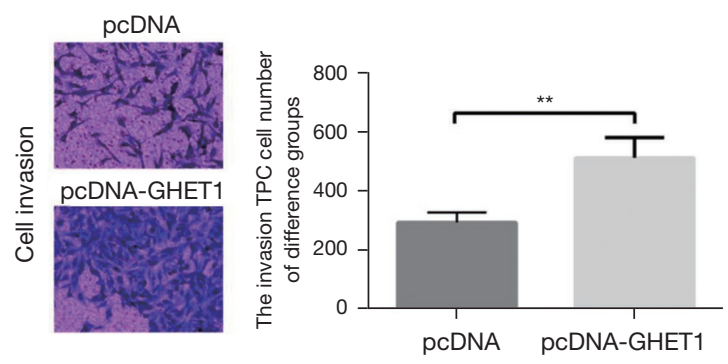

G

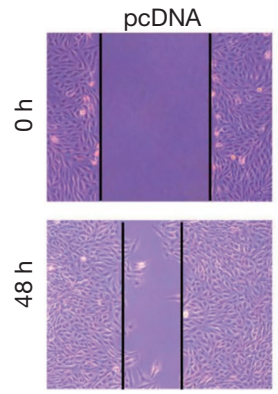

pCDNA-GHET1
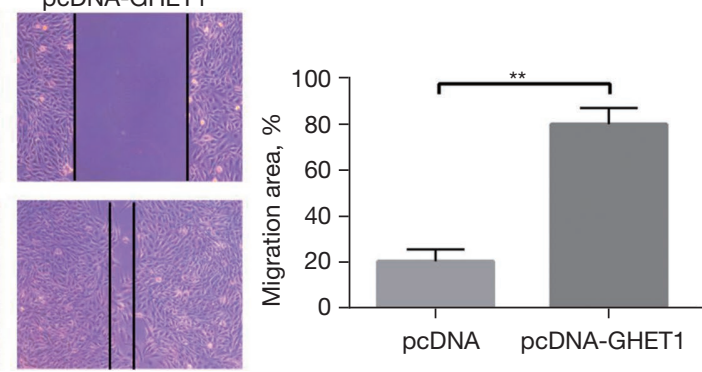

Figure 3 The influence of LncRNA GHET1 overexpression on TPC cell cell migration, apoptosis, , invasion cell cycle, and proliferation. (A) QRT-PCR results showing LncRNA GHET1 in TPC cells transfected with GHET1-overexpressing plasmid (pcDNA-GHET1) or GHET1-overexpressing plasmid (pcDNA-GHET1) pcDNA3.1 (pcDNA). (B) CCK-8 showing the influence of LncRNA GHET1 overexpression on TPC cell proliferation. (C) Colony formation assay results showing the effect of LncRNA GHET1 overexpression on the colony formation ability of TPC cells. Crystal violet staining. (D) Cell apoptosis and (E) cell cycle in TPC cells determined by flow cytometry. (F) Transwell showing the effect of LncRNA GHET1 overexpression on TPC cell invasion. Crystal violet staining, $\times 200$. (G) Wound-healing assay results demonstrating wound-healing rates in the overexpression groups and control groups in TPC cells. Crystal violet staining, $\times 100$. The data in vitro are on behalf of three independent experiments and expressed as the mean $\pm \mathrm{SD} .{ }^{* *}, \mathrm{P}<0.05$. 
tumors and may serve as a useful prognostic biomarker in Chinese cancer patients (19). In addition, an elevated LncRNA GHET1 expression has been significantly related to a more advanced clinical stage, earlier lymph node metastasis, earlier distant metastasis, and larger tumor size (20). Moreover, LncRNA GHET1 has been revealed to have a novel role in head and neck tumors (18). The results showed that LncRNA GHET1 might represent a new therapeutic target (21). LncRNA GHET1 also has a vital role in the development of pre-eclampsia; LncRNA GHET1 overexpression improved pre-eclampsia by suppressing E-cadherin and stimulating vimentin and fibronectin (22). Also, increased LncRNA GHET1 expression has been found in bladder cancer and has been associated with tumor TNM status, poor survival rates, and tumor size (23). LncRNA GHET1 may also act as an oncogenic LncRNA in cervical cancer (24). Furthermore, several studies have reported that LncRNA GHET1 may predict poor prognosis in carcinomas, including gastric cancer $(17,25,26)$, bladder tumor (27), colorectal tumor (28), hepatocellular cancer $(29,30)$, esophageal cancer (31), osteosarcoma (32), non-small cell lung tumor $(33,34)$, head and neck tumor (21), breast tumor (35), and prostate cancer (36). However, the correlation between LncRNA GHET1 and thyroid cancer remains unclear.

In our previous research, we reported that LncRNA GHET1 might be a target in initial TC progression. This study showed that LncRNA GHET1 was upregulated in thyroid cancer tissues and cell lines. Increased LncRNA GHET1 expression was significantly associated with TNM stage, lymph node metastasis, and poor outcomes in TC patients. Moreover, silencing LncRNA GHET1 can suppress the proliferation, migration, and invasion of human TPC and BCPAP cells and promote cell apoptosis in vitro.

In hepatocellular tumors, LncRNA GHET1 silencing has been shown to suppress the proliferation, invasion, migration, and EMT of HepG2 and SMMC-7721 cell lines in vitro (29). In pancreatic cancer, inhibiting the expression of LncRNA GHET1 inhibited the proliferation capacity, promoted apoptosis, and stopped the cell cycle in the G0/G1 phase (11). In this study, we proved that LncRNA GHET1 is overexpressed in PTC cell lines (TPC and BCPAP cells) and that silencing LncRNA GHET1 inhibited the invasion, migration, and proliferation of thyroid cells in vitro. Furthermore, to ascertain the molecular mechanism, we performed flow cytometry experiments that demonstrated LncRNA GHET1 silencing induced cell cycle arrest and promoted apoptosis in thyroid cancer cells in vitro.

The present study provides proof that upregulated LncRNA GHET1 promotes the progression of TC by promoting the proliferation and migration of thyroid cancer cells. Our analysis established that a high LncRNA GHET1 expression was significantly associated with TNM stage and lymph node metastasis. However, further studies are needed to verify these results and reveal the underlying molecular mechanism of LncRNA GHET1 in thyroid cancer. Besides some experiments in vivo should be increased. Papillary thyroid cancer is known to have a good prognosis. Our study subjects have a very good prognosis. More studies about the prognostic value of LncRNA GHET1 in thyroid cancer are needed. In addition, LncRNA GHET1 has remarkable tumor specificity and decreased systemic toxicity compared with cytotoxic chemotherapy. Therefore, LncRNA GHET1 could be used as a potential and viable therapeutic target.

In this study, we investigated the role of LncRNA GHET1 in thyroid cancer. This study demonstrated for the first time that LncRNA GHET1 expression was upregulated in thyroid cancer tissues. These results indicate that LncRNA GHET1 affects the progression of thyroid cancer.

In conclusion, this study found that LncRNA GHET1 is a promoting tumor factor in thyroid cancer. The knockdown of LncRNA GHET1 can suppress the biological activity of TPC and BCPAP thyroid cancer cell lines. These results suggest that LncRNA GHET1 could be used as a molecular marker of thyroid cancer progression and a molecular target for therapy.

\section{Acknowledgments}

Funding: This study was supported by Hebei Science and Technology Planning (grant No. 16967788D) and the Hebei Health Department Project (grant No. 20201133).

\section{Footnote}

Reporting Checklist: The authors have completed the MDAR reporting checklist. Available at https://dx.doi. org/10.21037/tcr-21-1615

Data Sharing Statement: Available at https://dx.doi. org/10.21037/tcr-21-1615

Conflicts of Interest: All authors have completed the ICMJE uniform disclosure form (available at https://dx.doi. 
org/10.21037/tcr-21-1615). The authors have no conflicts of interest to declare.

Ethical Statement: The authors are accountable for all aspects of the work in ensuring that questions related to the accuracy or integrity of any part of the work are appropriately investigated and resolved. All patients provided written informed consent, and this study was approved by the Ethics Committee of the Hebei Provincial Tumor Hospital (Ethics Review Number: 2020KS017). All procedures performed in this study involving human participants were in accordance with the Declaration of Helsinki (as revised in 2013).

Open Access Statement: This is an Open Access article distributed in accordance with the Creative Commons Attribution-NonCommercial-NoDerivs 4.0 International License (CC BY-NC-ND 4.0), which permits the noncommercial replication and distribution of the article with the strict proviso that no changes or edits are made and the original work is properly cited (including links to both the formal publication through the relevant DOI and the license). See: https://creativecommons.org/licenses/by-nc-nd/4.0/.

\section{References}

1. Jendrzejewski J, Thomas A, Liyanarachchi S, et al. PTCSC3 Is Involved in Papillary Thyroid Carcinoma Development by Modulating S100A4 Gene Expression. J Clin Endocrinol Metab 2015;100:E1370-7.

2. Zhao JJ, Hao S, Wang LL, et al. Long non-coding RNA ANRIL promotes the invasion and metastasis of thyroid cancer cells through TGF- $\beta /$ Smad signaling pathway. Oncotarget 2016;7:57903-18.

3. Jendrzejewski J, He H, Radomska HS, et al. The polymorphism rs 944289 predisposes to papillary thyroid carcinoma through a large intergenic noncoding RNA gene of tumor suppressor type. Proc Natl Acad Sci U S A 2012;109:8646-51.

4. Cui M, Chang Y, Du W, et al. Upregulation of lncRNAATB by Transforming Growth Factor $\beta 1$ (TGF- $\beta 1$ ) Promotes Migration and Invasion of Papillary Thyroid Carcinoma Cells. Med Sci Monit 2018;24:5152-8.

5. Wang S, Luo J, Zhong Z, et al. Treatment and prognosis for patients with differentiated thyroid carcinoma bone metastases. Transl Cancer Res 2020;9:972-82.

6. Naoum GE, Morkos M, Kim B, et al. Novel targeted therapies and immunotherapy for advanced thyroid cancers. Mol Cancer 2018;17:51.

7. Yan HX, Du J, Fu J, et al. Microarray-based differential expression profiling of long noncoding RNAs and messenger RNAs in formalin-fixed paraffin-embedded human papillary thyroid carcinoma samples. Transl Cancer Res 2019;8:439-51.

8. Zhang J, Zou S, Deng L. Gene Ontology-based function prediction of long non-coding RNAs using bi-random walk. BMC Med Genomics 2018;11:99.

9. Xia F, Chen Y, Jiang B, et al. Long Noncoding RNA HOXA-AS2 Promotes Papillary Thyroid Cancer Progression by Regulating miR-520c-3p/S100A4 Pathway. Cell Physiol Biochem 2018;50:1659-72.

10. Song JY, Sun SR, Dong F, et al. Predictive Value of BRAFV600E Mutation for Lymph Node Metastasis in Papillary Thyroid Cancer: A Meta-analysis. Curr Med Sci 2018;38:785-97.

11. Li J, Jiang X, Li Z, et al. Long noncoding RNA GHET1 in human cancer. Clin Chim Acta 2019;488:111-5.

12. Yang F, Xue X, Zheng L, et al. Long non-coding RNA GHET1 promotes gastric carcinoma cell proliferation by increasing c-Myc mRNA stability. FEBS J 2014;281:802-13.

13. Ji D, Zhong $X$, Jiang $X$, et al. The role of long non-coding RNA AFAP1-AS1 in human malignant tumors. Pathol Res Pract 2018;214:1524-31.

14. Li D, Li L, Chen X, et al. LncRNA GHET1 promotes osteoblast proliferation and differentiation by inhibiting PTEN. Panminerva Med 2019. [Epub ahead of print]. doi: 10.23736/S0031-0808.19.03701-7.

15. Zhang K, Li C, Liu J, et al. DNA methylation alterations as therapeutic prospects in thyroid cancer. J Endocrinol Invest 2019;42:363-70.

16. Ling W, Yangchun X, Wei W, et al. Knockdown of long non-coding RNA GHET1 suppresses cervical carcinoma in vitro and in vivo. Cancer Biomark 2020;28:21-32.

17. Sun W, Yang Y, Xu C, et al. Roles of long noncoding RNAs in gastric cancer and their clinical applications. J Cancer Res Clin Oncol 2016;142:2231-7.

18. Ge MH, Jiang LH, Wen QL, et al. Preliminary screening and analysis of metastasis-related lncRNA and coexpressed papillary thyroid carcinoma mRNA. Oncol Lett 2018;16:3715-25.

19. Song J, Chen X, Tian Q, et al. The Value of lncRNA GHET1 as a Prognostic Factor for Survival of Chinese Cancer Outcome: A Meta-Analysis. Dis Markers 2019;2019:5824190.

20. Jiang YF, Zhang HY, Ke J, et al. Overexpression of LncRNA GHET1 predicts an unfavourable survival and 
clinical parameters of patients in various cancers. J Cell Mol Med 2019;23:4891-9.

21. Liu $\mathrm{H}, \mathrm{Wu} \mathrm{Y}$. Long non-coding RNA gastric carcinoma highly expressed transcript 1 promotes cell proliferation and invasion in human head and neck cancer. Oncol Lett 2018;15:6941-6.

22. Li S, Liu J, Kong F, et al. lncRNA GHET1 has effects in development of pre-eclampsia. J Cell Biochem 2019;120:12647-52.

23. Li B, Xie D, Zhang H. Long non-coding RNA GHET1 contributes to chemotherapeutic resistance to Gemcitabine in bladder cancer. Cancer Chemother Pharmacol 2019;84:187-94.

24. Zhang Q, Zhang Y, Wang Y. GHET1 acts as a prognostic indicator and functions as an oncogenic lncRNA in cervical cancer. Biosci Rep 2019;39:BSR20182506.

25. Huang $\mathrm{H}$, Liao $W$, Zhu X, et al. Knockdown of long noncoding RNA GHET1 inhibits cell activation of gastric cancer. Biomed Pharmacother 2017;92:562-8.

26. Xia Y, Yan Z, Wan Y, et al. Knockdown of long noncoding RNA GHET1 inhibits cell-cycle progression and invasion of gastric cancer cells. Mol Med Rep 2018;18:3375-81.

27. Li LJ, Zhu JL, Bao WS, et al. Long noncoding RNA GHET1 promotes the development of bladder cancer. Int J Clin Exp Pathol 2014;7:7196-205.

28. Zhou J, Li X, Wu M, et al. Knockdown of Long Noncoding RNA GHET1 Inhibits Cell Proliferation and Invasion of Colorectal Cancer. Oncol Res 2016;23:303-9.

29. Jin L, He Y, Tang S, et al. LncRNA GHET1 predicts poor prognosis in hepatocellular carcinoma and promotes cell proliferation by silencing KLF2. J Cell Physiol

Cite this article as: Liu Y, Shi P, Fang H, Zhao Z, Yang F, Zhang J, Jing S, Geng C. Long non-coding RNA GHET1 promotes thyroid cancer cell proliferation and invasion. Transl Cancer Res 2021;10(9):4148-4157. doi: 10.21037/tcr-21-1615
2018;233:4726-34.

30. Ding G, Li W, Liu J, et al. LncRNA GHET1 activated by $\mathrm{H} 3 \mathrm{~K} 27$ acetylation promotes cell tumorigenesis through regulating ATF1 in hepatocellular carcinoma. Biomed Pharmacother 2017;94:326-31.

31. Liu H, Zhen Q, Fan Y. LncRNA GHET1 promotes esophageal squamous cell carcinoma cells proliferation and invasion via induction of EMT. Int J Biol Markers 2017;32:e403-8.

32. Yang $W$, Shan Z, Zhou X, et al. Knockdown of lncRNA GHET1 inhibits osteosarcoma cells proliferation, invasion, migration and EMT in vitro and in vivo. Cancer Biomark 2018;23:589-601.

33. Guan ZB, Cao YS, Li Y, et al. Knockdown of lncRNA GHET1 suppresses cell proliferation, invasion and LATS1/YAP pathway in non small cell lung cancer. Cancer Biomark 2018;21:557-63.

34. Shen QM, Wang HY, Xu S. LncRNA GHET1 predicts a poor prognosis of the patients with non-small cell lung cancer. Eur Rev Med Pharmacol Sci 2018;22:2328-33.

35. Song R, Zhang J, Huang J, et al. Long non-coding RNA GHET1 promotes human breast cancer cell proliferation, invasion and migration via affecting epithelial mesenchymal transition. Cancer Biomark 2018;22:565-73.

36. Zhu Y, Tong Y, Wu J, et al. Knockdown of LncRNA GHET1 suppresses prostate cancer cell proliferation by inhibiting HIF-1 $\alpha$ /Notch-1 signaling pathway via KLF2. Biofactors 2019;45:364-73.

(English Language Editor: D. Fitzgerald) 